\title{
The strain-rate effect of engineering materials and its unified model
}

\section{Abstract}

The test data of metals, brittle materials and polymers in high, medium and low strain-rate range were summarized. It was found that the dynamic strength or yield stress of these materials was not sensitive to strain-rate in the low and high strain-rate range (corresponding to weak sensitivity area and saturated zone). But in the medium strain-rate range (strong sensitivity area), the dynamic strength or yield stress of these materials was extremely sensitive to strain-rate. The strain-rate effect function in classic Johnson-Cook constitutive model could not describe these phenomena in the range of low to high strain-rate. Therefore, the strain-rate effect function in classic Johnson-Cook constitutive model has been modified to a strain-rate effect expression which is suitable for different materials. This new model can better describe the phenomena of the weak sensitivity area, strong sensitivity area and saturated zone. With this model, the fitted curves are in good agreement with the test data, and the parameters are different for different materials. Finally, the fitting parameters are given.

\section{Keywords}

Solid mechanics, strain-rate effect, Johnson-Cook, constitutive model, parameter

\section{INTRODUCTION}

The dynamic mechanical property of materials under impact loads is a hot research topic of the impact dynamics. The difference of the material behavior under impact loading and quasi-static loading is that the dynamic strength or yield stress increases as the strain-rate increases. Fig.1 illustrates the general features of dynamic strength or yield stress dependence on strain-rate (Qi et al., 2009). In the lower strain-rate range, the material dynamic strength or yield stress increases slowly with increasing strain-rate (identified as Regime I, weak sensitivity area). Generally, the method of 
hydraulic loading machine or microcomputer controlled material testing machine is used in this area. As shown in Figure 1, Regime II (named as a strong sensitivity area) is characterized by a rapid material strength or yield stress increase when the strain-rate exceeds a threshold level. Common test methods for this strain-rate range are split Hopkinson pressure bar (SHPB) test and expanding ring technology. At very high strain-rate in Regime III (referred to as a saturation zone), the dynamic strength or yield stress dependence becomes weak again, having similarity to Regime I. Common test methods for Regime III are plate impact test and one-stage light gas gun technology. Many studies focused on the dynamic behavior of materials in the lower strain-rate and moderate strain-rate range (weak sensitivity area and strong sensitivity area) [Wang and Wang ,2011; Ren et al., 2010; Pang et al., 2011; Fu et al., 2010; Zhou and John, 2001], and obtained a general conclusion that with the increase of strain-rate, the dynamic strength or yield stress of materials is also increased. So the problem is that when the strain-rate continues to increase, will the strain-rate effect be continuing to increase? It obviously will obtain infinite strain-rate dependence. The solution for this problem given by some researchers ( $\mathrm{Tu}$ and Lu, 2009; Zhou et al., 2006) was that when the magnitude of the strain-rate was beyond $10^{3} \mathrm{~s}^{-1}$, the dynamic strength or yield stress of materials no longer increased with the increase of strain-rate, and the strain-rate dependence is capped as that at $10^{3} \mathrm{~s}^{-1}$. But they did not give explanations. Alves (2000) compared tensile flow stress data for mild steel under various strain-rates with conventional Cowper-Symonds equation, and Marais et al. (2004) compared the dynamic compressive data of mild steel and copper based on SHPB tests with the conventional Johnson-Cook (JC) and CS models. They found that the correlation is not good unless the modification of the CS equation is made or the coefficients of these conventional constitutive equations are changed. In this paper, through extensive literature study, the test data of metals, brittle materials and polymers in high, medium and low strain-rate range were summarized. The strain-rate effect function in classic JC constitutive model (Johnson and Cook, 1983) was modified to a strain-rate effect expression suitable for different materials in a wide range of strain-rate. Fitted curves using the modified model are in good agreement with the test data, and these fitted curves all present a three-regime phenomenon. Parameters of the fitted curves are different for different materials. Finally, the fitted parameters are given.

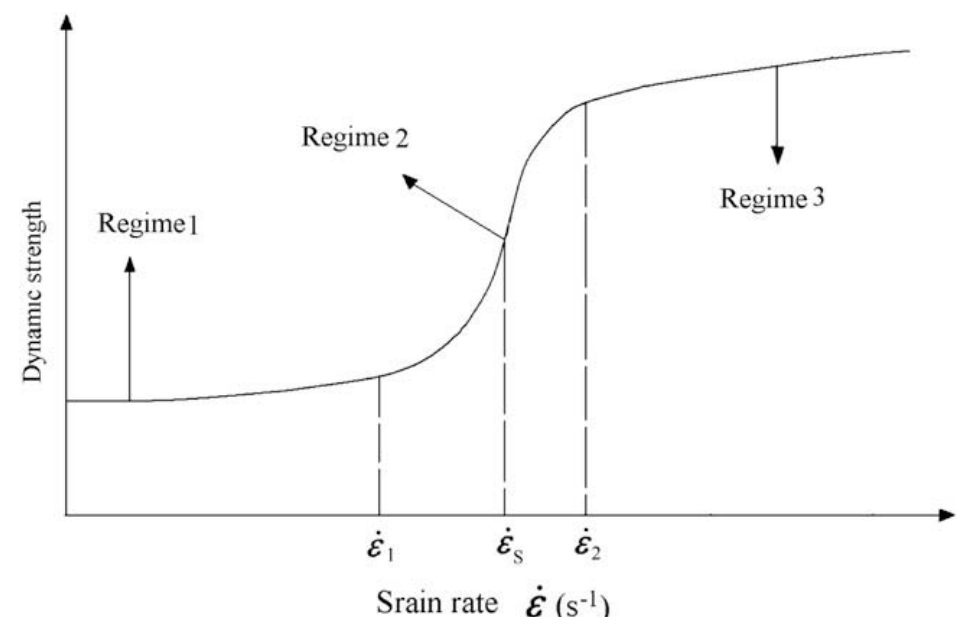

Figure 1 Dependence of dynamical strength/yield stress on strain-rates of engineering materials (Qi et al., 2009) 


\section{METALS}

\subsection{5 aluminum}

Zhang et al. (2011) performed experiments in a wide range of strain-rate to study the dynamic response of 7075 aluminum from quasi-static uniaxial stress to uniaxial strain flyer impact. The quasistatic tests of 7075 aluminum were carried on a microcomputer controlled material testing machine. Compressive dynamic tests in moderate strain-rate range were carried on a $\Phi 14.5 \mathrm{~mm}$ SHPB set-up. Uniaxial strain flyer impact tests were performed by the use of $\Phi 57 \mathrm{~mm}$ light gas gun combined with single point and double sensitivity VISAR testing system. Based on the stress-strain curves at various strain-rates, dynamic increase factor (DIF), defined as the ratio of dynamic strength or yield stress to the quasi-static strength or yield stress of materials, versus the common logarithmic of strain-rates can be obtained.

As shown in Figure 2, at low strain-rates (approximately less than $10^{2} \mathrm{~s}^{-1}$ ), the yield stress of 7075 aluminum is not sensitive to strain-rate, and strain-rate sensitivity is weak. However, at moderate strain-rates, the yield stress of 7075 aluminum is very sensitive to the strain-rate in a small range near $1.6 \times 10^{3} \mathrm{~s}^{-1}$. When the strain-rate is greater than $10^{4} \mathrm{~s}^{-1}$, the strain-rate dependence of 7075 aluminum becomes saturated, and the yield stress no longer increases obviously as the strainrate increases.

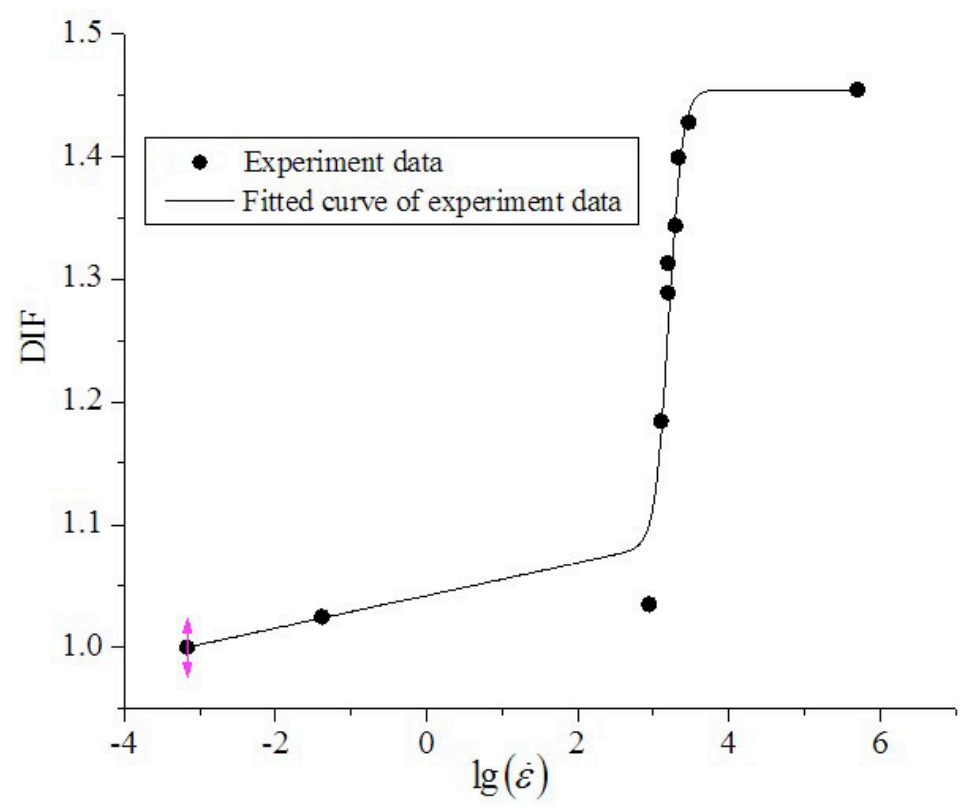

Figure 2 The relationship between DIF and common logarithmic of strain-rate for 7075 aluminum 


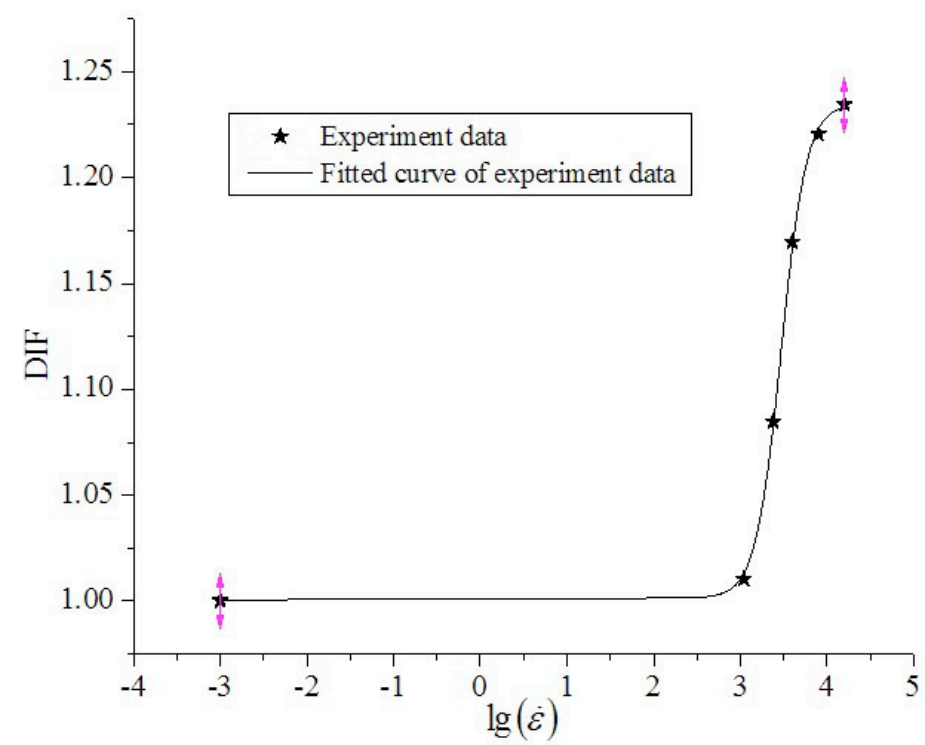

Figure 3 The relationship between DIF and common logarithmic of strain-rate for stainless steel

\subsection{Stainless steel}

Zhang et al. (2012) studied the dynamic mechanical properties of stainless steel at high strain-rates. Quasi-static tests were carried on an electronic universal testing machine with $\Phi 5 \mathrm{~mm} \times 5 \mathrm{~mm}$ cylindrical samples, the strain-rate is kept as $0.001 \mathrm{~s}^{-1}$ through controlling the loading rate. Dynamic tests at five different strain-rates of $1100 \mathrm{~s}^{-1}, 2400 \mathrm{~s}^{-1}, 4000 \mathrm{~s}^{-1}, 8000 \mathrm{~s}^{-1}$ and $16000 \mathrm{~s}^{-1}$ were conducted. Among them, compression dynamic tests at strain-rate of $1100 \mathrm{~s}^{-1}$ and $2400 \mathrm{~s}^{-1}$ were on $\Phi 12.7 \mathrm{~mm}$ SHPB set-up with $\Phi 5 \mathrm{~mm} \times 5 \mathrm{~mm}$ cylindrical samples. $\Phi 2 \mathrm{~mm} \times 2 \mathrm{~mm}$ cylindrical samples used for strain-rates of $4000 \mathrm{~s}^{-1}$ and $8000 \mathrm{~s}^{-1}$, and $\Phi 2 \mathrm{~mm} \times 1 \mathrm{~mm}$ cylindrical samples employed for the strain-rate of $16000 \mathrm{~s}^{-1}$, were tested on a $\Phi 5 \mathrm{~mm}$ miniature SHPB set-up.

Based on the above experiments, the relationship between DIF and the logarithmic of strainrates was obtained, as shown in Figure 3. It is shown that the relationship between DIF and the logarithmic of strain-rates exhibits obvious three regimes. The strain-rate sensitivity is weak in the strain-rate range of $0.001 \mathrm{~s}^{-1}$ to $1100 \mathrm{~s}^{-1}$. In the strain-rate range of $1100 \mathrm{~s}^{-1}$ to $8000 \mathrm{~s}^{-1}$, the strainrate sensitivity becomes strong. While with the increase of the strain-rate from $8000 \mathrm{~s}^{-1}$ to $16000 \mathrm{~s}^{-1}$, DIF increases slowly and tends to a saturation zone.

\section{BRITTLE MATERIALS}

\subsection{Limestone and concrete}

Limestone and concrete were also observed to show certain saturation phenomenon of strain-rate dependence, as shown in Figure 4. The test data of limestone and concrete in low and medium 
strain-rate range were obtained from Green and Perkins (1972) and Le Nard and Bailly (2000), respectively. Under high strain-rates, the test data of limestone and concrete were obtained from uniaxial strain tests by Brace \& Jones (1971) and Gupta \& Seaman (1978), respectively. Comparing experimental data at different strain-rates indicates that the limestone and concrete show obvious strain-rate sensitivity. From DIF versus the common logarithmic of strain-rate relationship of limestone and concrete in Figure 4, the DIF of limestone and concrete also shows obvious three regimes with the increase of strain-rate, viz. weak sensitivity area, strong sensitivity area and saturated zone.

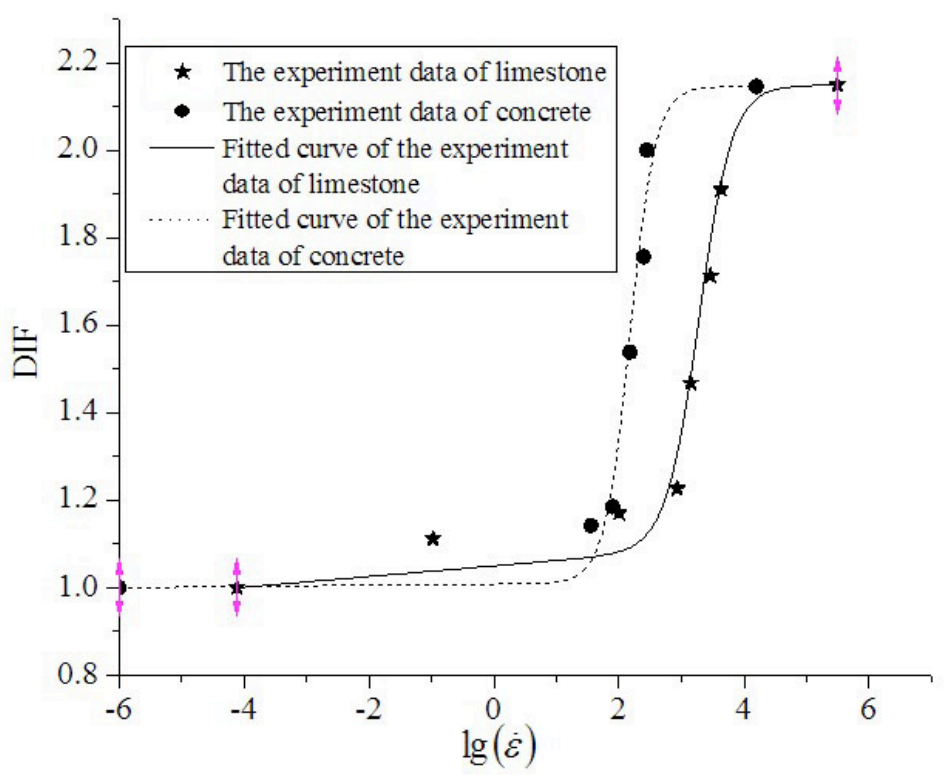

Figure 4 The relationship between DIF and common logarithmic of strain-rate for limestone and concrete

\subsection{Domolite and granodiorite}

Qi \& Qian (2003) studied the physical mechanism of the dynamic strength dependence on strainrate for rock-like materials. They found that, in the range of low strain-rate, the strain-rate dependence of the dynamic strength was controlled by thermoactivational mechanism. With the increase of strain-rate, the macro-viscosity mechanism arises, and gradually becomes dominated. In high strainrate region, the inertial effect becomes significant, and the growth of defects of a wide range of sizes will be initiated simultaneously, and then the thermoactivational rupture of atomic bonds will induce in the area without defects. Thus, the strain-rate sensitivity of dynamic strength was considered as the competition result between the coexisting thermoactivational and thermo-viscosity mechanism, which played dominated role in different strain-rate regions. A unified model of competition between thermoactivational and thermo-viscosity mechanism was given.

The relation between DIF and the common logarithmic of strain-rate of dolomite and granodiorite is shown in Figure 5, where the two endpoints are experimental data from Grady (1998), while 
others were predicted data by the model in Qi \& Qian (2003). The fitted curves using the following proposed model are in good agreement with the data.

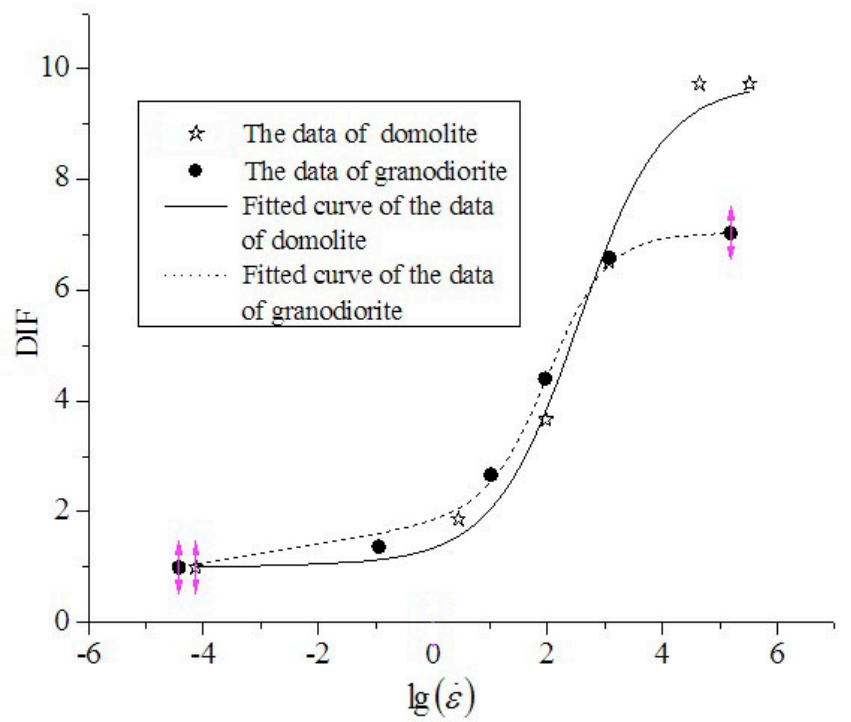

Figure 5 The relationship between DIF and common logarithmic of strain-rate for domolite and granodiorite

\subsection{Silicon carbide and alumina}

Qi \& Qian (2003) studied silicon carbide and alumina using the method as descried in Section 3.2. The fitted curves using the following proposed model are in good agreement with the experimental data from Grady (1998) and predicted data by the model in Qi \& Qian (2003), as shown in Figure 6 . The fitted curves have the phenomenon of three regimes.

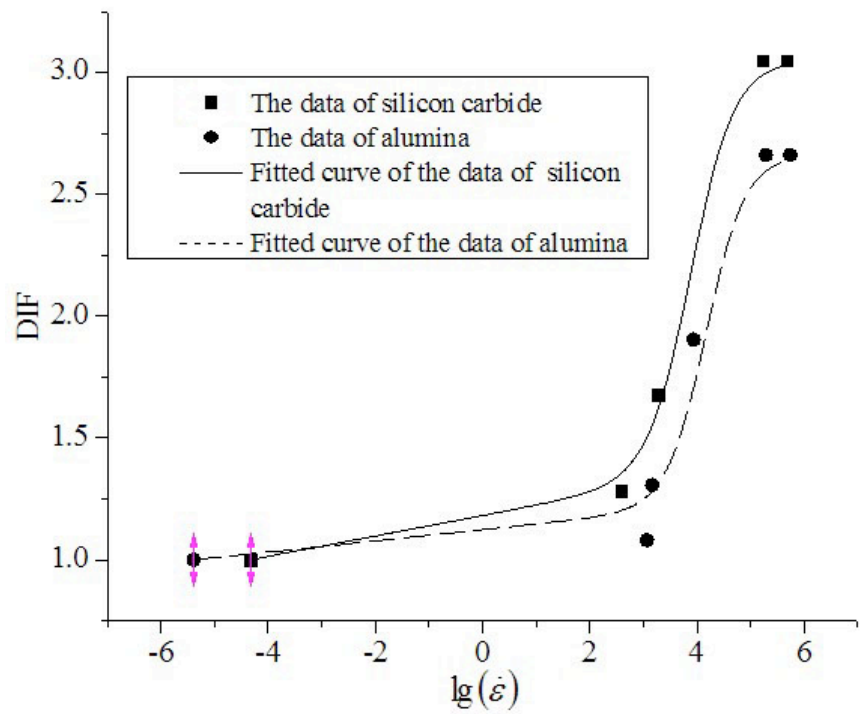

Figure 6 The relationship between DIF and common logarithmic of strain-rate for silicon carbide and alumina 


\section{POLYMERS}

\subsection{PC}

$\mathrm{Hu}$ et al. (2006) studied the impact compression performance of polycarbonate (PC) by the use of $\Phi 25 \mathrm{~mm}$ SHPB device. The quasi-static tests were performed on the MTS810 fatigue testing machine. The size of PC samples for these tests is $\Phi 15 \mathrm{~mm} \times 8 \mathrm{~mm}$. Test results showed that the deformation mechanism of PC under impact loading is obviously different from that under quasistatic loading. The relation of the DIF and the common logarithmic of strain-rate for PC is shown in Figure 7. It is observed that the strain-rate sensitivity of PC is weak under lower strain-rates, PC shows strong strain-rate sensitivity at moderate strain-rates, and the DIF increases slowly with further increase of strain-rate and tended to saturation gradually.

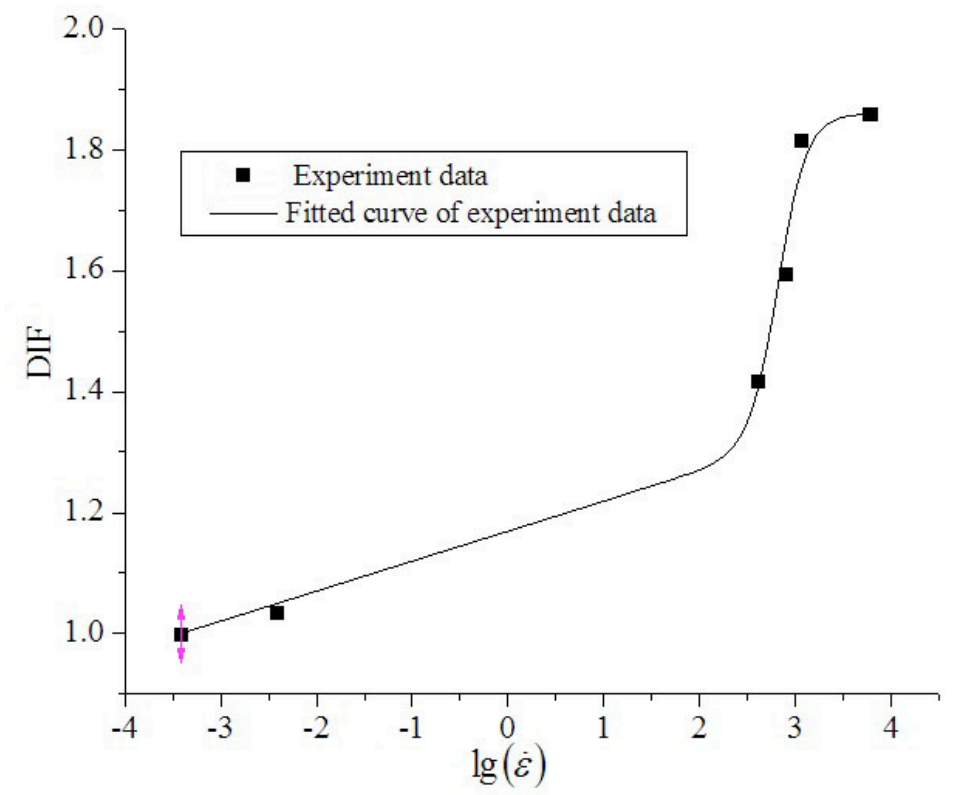

Figure 7 The relationship between DIF and common logarithmic of strain-rate for PC

Sato et al. (2008) determined the stress-strain relationships of PC under uniaxial strain state over a very wide strain-rate range of $10^{-4} \mathrm{~s}^{-1}$ to $10^{7} \mathrm{~s}^{-1}$. Plate impact tests combined with a newly modified unsteady wave sensing system, drop-weight tests, and quasi-static tests using universal and Instron testing machines were used for the high strain-rate $\left(10^{7} \mathrm{~s}^{-1}\right)$, medium strain-rate $\left(10^{2} \mathrm{~s}^{-1}\right)$ and low strain-rate $\left(10^{-4} \mathrm{~s}^{-1}\right)$ tests, respectively. An empirical formula of the relationship between stress and strain-rate was fitted according to the experimental data. It was found that the relationship between stress and strain-rate under uniaxial strain condition is nearly linear over the wide range of strain-rate. 


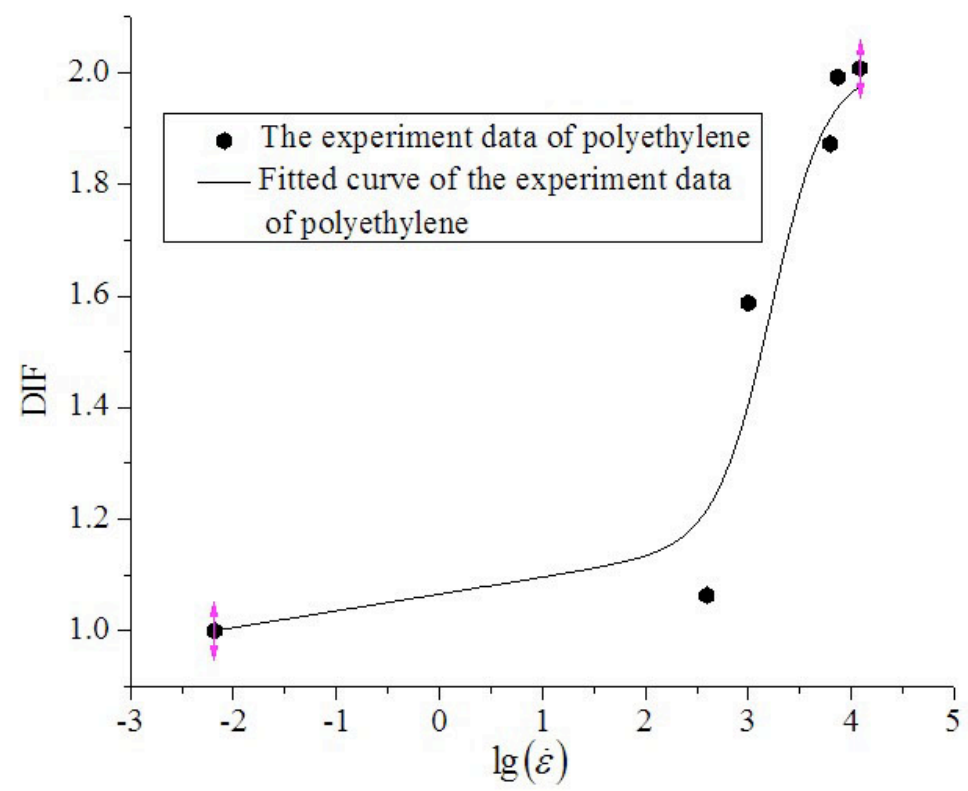

Figure 8 The relationship between DIF and common logarithmic of strain-rate for polyethylene.

\subsection{Polyethylene}

Al-Maliky and Parry (1994) studied the high-density polyethylene at very high tensile strain-rates (above $10^{4} \mathrm{~s}^{-1}$ ) using an expanding ring technique, which involves placing a thin ring of polymer as a sliding fit around a hollow thick-walled cylinder. A blast wave generated by an exploding wire produces a pressure pulse acting on the internal wall of the cylinder. This pulse propagates through the cylinder wall and is partially transferred into the ring. The ring moves virtually instantaneously at a high velocity away from the cylinder and then decelerates under almost uniaxial tension as a result of the hoop stress. As can be seen in Figure 8, the relationship between DIF and the common logarithmic of strain-rate for polyethylene also exhibits the phenomenon of three regimes.

\subsection{Resin}

Gerlach et al. (2008) studied the mechanical properties of RTM-6 resin under impact loading. A novel pulse shaping technique and specimen design were employed for performing SHPB tensile and compression tests on RTM-6 resin. Test results are summarized in Figure 9. It is observed that the relationship between DIF and the common logarithmic of strain-rate obtained from either SHPB compression tests or tensile tests exhibits the phenomenon of three regimes. At lower strain-rates, the resin shows weak strain-rate dependence. When the common logarithmic of strain-rate is in the range of 3 to 3.5, the resin shows strong strain-rate dependence. Afterwards, DIF increases slowly with increasing strain-rate and gradually tends to saturation. 


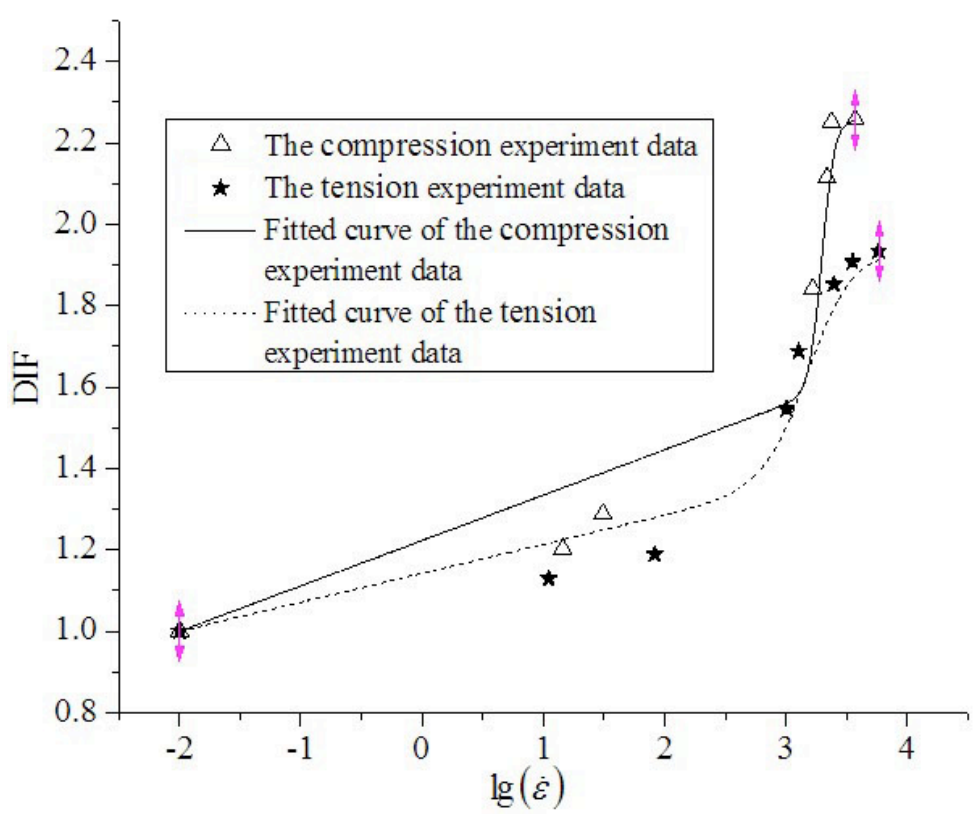

Figure 9 The relationship between DIF and common logarithmic of strain-rate for RTM- 6 resin

Table 1 The magnitude of two transition strain-rates for engineering materials.

\begin{tabular}{cccc}
\hline \hline & Metals & Brittle materials & Polymers \\
\hline The first turning point & $10^{3} \mathrm{~s}^{-1}$ & $10^{2} \mathrm{~s}^{-1}$ & $10^{2.5} \mathrm{~s}^{-1}$ \\
\cline { 1 - 1 } The second turning point & $10^{4} \mathrm{~s}^{-1}$ & $10^{4} \mathrm{~s}^{-1}$ & $10^{3.5} \mathrm{~s}^{-1}$ \\
\hline \hline
\end{tabular}

\section{RESULTS AND DISCUSSION}

The data of metals, brittle materials and polymers at high, medium and low strain-rates were summarized (Figures 2-9). It is found that they have a common characteristic of having three regimes and two transition strain-rates. But the magnitude of transition strain-rates is different for different types of materials. It may be due to the difference of the strength or yield stress for various types of materials, viz. the deformation physical mechanisms of metals, brittle materials and polymers are different. According to the data shown in Figures 2-9, the magnitude of transition strain-rates for various types of materials is estimated and summarized, as shown in Table 1, where the magnitude of transition strain-rates is an approximate value rather than an exact value.

Based on the data summarized in Figures 2-9, the mathematical expression for describing the strain-rate effect in the Johnson-Cook constitutive model is modified. The relationship between DIF and the common logarithmic of strain-rate is expressed as, 


$$
\mathrm{DIF}=\left(1+A \varepsilon^{n}\right)\left\{\mathrm{DIF}_{\text {max }}-\frac{\mathrm{DIF}_{\text {max }}-\left[1+B\left(\lg \dot{\varepsilon}-\lg \dot{\varepsilon}_{0}\right)\right]}{1+\exp \left[C\left(\lg \dot{\varepsilon}-\lg \dot{\varepsilon}_{c}\right)\right]}\right\}\left(1-T^{* m}\right)
$$

where $A, B, C, m$ and $n$ are fitted parameters; $\mathrm{DIF}_{\max }$ is the maximum DIF in the saturated zone; $e$ is the strain corresponding to the maximum strength or yield stress in the saturated zone; $\dot{\varepsilon}$ is the current testing strain-rate; $\dot{\varepsilon}_{0}$ is the reference strain-rate, which is the strain-rate under quasistatic tests; $\dot{\varepsilon}_{c}$ was the strain-rate corresponding to the midpoint of strong strain-rate sensitivity regime, determining the location of this regime; $T^{*}=\left(T-T_{r}\right) /\left(T_{m}-T_{r}\right), T$ is the current testing temperature, $T_{r}$ is room temperature, and $T_{m}$ is the melting temperature of materials.

It is found that data shown in Figures 2-9 can be well fitted by Eq.(1). The values of $R^{2}$ for the fitted curves to those data in Figures 2-9 are summarized in Table 2. Fitted parameters of Eq.(1) for different materials are shown in Table 3. Since those data shown in Figures 2-9 are obtained at room temperature, $T^{*}$ is zero. Additionally, experimental data of engineering materials under high strain-rates and high temperatures are lacked. Accordingly, the parameter of $m$ is not fitted. Therefore, further validation is required for Eq.(1) under low and high temperatures.

Table 2 The values of $R^{2}$ for fitted curves to data.

\begin{tabular}{cccccc}
\hline \hline & \multicolumn{1}{c}{$R^{2}$} & $R^{2}$ & & $R^{2}$ \\
\hline 7075 aluminum & 0.953 & stainless steel & 0.999 & limestone & 0.961 \\
concrete & 0.951 & domolite & 0.982 & granodiorite & 0.993 \\
silicon carbide & 0.986 & alumina & 0.934 & PC & 0.977 \\
polyethylene & 0.849 & resin (compression) & 0.934 & resin (tension) & 0.938 \\
\hline \hline
\end{tabular}

Table 3 The fitted parameters in Eq.(1) for different materials.

\begin{tabular}{ccccc}
\hline \hline & $A$ & $B$ & $C$ & $n$ \\
\hline 7075 aluminum & 1.48 & 0.01 & 10.86 & 1.73 \\
stainless steel & 0.02 & 0.00016 & 6.92 & 1.57 \\
limestone & -8.93 & 0.012 & 3.81 & 1566.99 \\
concrete & -1.90 & 0.0014 & 4.96 & 3161.18 \\
domolite & -5.50 & 0.019 & 1.35 & 99.72 \\
granodiorite & -5.94 & 0.17 & 1.93 & 110.34 \\
Silicon carbide & -7.30 & 0.042 & 2.49 & 143.82 \\
& & & & \\
alumina & -1.59 & 0.023 & 2.75 & 310.64 \\
PC & -7.67 & 0.05 & 6.67 & 172.76 \\
polyethylene & 2.09 & 0.03 & 3.75 & 119.45 \\
resin (compression) & -45.6 & 0.11 & 20.71 & 18.00 \\
resin (tension) & 2.55 & 0.071 & 5.55 & 30.28 \\
\hline \hline
\end{tabular}


From Table 3, it is observed that the fitted parameters of Eq.(1) for different materials are different. However, among the values of $A, B, C$ and $n$, there are similarities among these parameters. For the parameter of $A$, metals have positive values, and brittle materials have negative values, while the sign of $A$ for polymers is uncertain. The value of $B$ for all materials is very small. In the fitting process, it is found that the fitted curves agree well with data points, even different values of the strain-rate corresponding to the midpoint of the strong strain-rate sensitivity regime, $\dot{\varepsilon}_{c}$, are used.

In general, constitutive models based on microscopic physical mechanisms are superior to empirically phenomenological constitutive models. But the intrinsic mechanisms of materials are complex and diverse over a wide range of strain-rates (e.g. $10^{-4} \mathrm{~s}^{-1}$ to $10^{6} \mathrm{~s}^{-1}$ ), which can not be well expressed in a single material model. Thus, until today, semi-theoretical material models still have great advantage in practical applications.

\section{CONCLUSIONS}

A large number of theoretical, experimental and numerical simulation studies have been done on the strain-rate effect of different materials under dynamic loading. It is found that there are two transition strain-rates for the strength or yield stress enhancement of these materials. When the strain-rate is over the first transition strain-rate, the strength or yield stress increases significantly with increasing strain-rate. And when the strain-rate is over the second transition strain-rate, the strength or yield stress increases slowly and tends to saturation with increasing strain-rate. The trend of DIF versus the common logarithmic of strain-rate can be divided into three regimes, viz. weak sensitivity area, strong sensitivity area and saturated zone. From the summarized data of metals, brittle materials and polymers in this study, it could be found that no matter what type of materials, such a common phenomenon of three regimes exists. For this phenomenon, a unified mathematical expression is proposed to describe the relationship of DIF and the common logarithmic of strain-rate. Fitted parameters of the expression are different for various materials as their dynamic mechanical properties are different. Fitted curves based on the proposed expression are in good agreement with data from various references. The expression for strain-rate dependence proposed in this study is relatively simple and feasible for practical applications over a wide range of strain-rates.

Acknowledgements This work is supported by the Scientific Research Fund of Sichuan Provincial Education Department (10zd1012), and Excellent Project Funding from Ministry of Human Resources and Social Security for Returned Overseas Students (11zs0103).

\section{References}

Al-Maliky, N. and Parry, D.J., (1994). Measurements of high strain rate properties of polymers using an expanding ring method. 4th International Conference on Mechanical and Physical Behavior of Materials under Dynamic Loading, 71-76. 
Alves, M. (2000). Material constitutive law for large strains and strain rates. Journal of Engineering Mechanics $126: 215-218$

Brace, W.F. and Jones, A.H., (1971). Comparison of uniaxial deformation in shock and static loading of three rocks. Journal of Geophysical Research 76: 4913-4921.

Fu, S.Q., Wang, Y. and Wang, Y., (2009). High strain-rate tensile experiment on polycarbonate bar. Journal of Experimental Mechanics 24: 202-206.

Gerlach, R., Siviour, C.R. and Petrinic, N., et al., (2008). Experimental characterization and constitutive modeling of RTM-6 resin under impact loading. Polymer 49: 2728-2737.

Grady, D.E., (1998). Shock wave properties of brittle solid. Mechanics of Materials 29: 181-203.

Green, S.J. and Perkins, R.D., (1972). Uniaxial compression tests at strain rates from 10-4/s-104/s on three geologic materials. In: Gray KE, editor. Proceedings of the 10th US rock mechanics symposium. New York: American Institute of Mining and Metallurgical Petroleum Engineering, 35-54.

Gupta, Y.M. and Seaman, L., (1978). Local response of reinforced concrete to missile impact. Nuclear Engineering and Design 45: 507-514

Hu, W.J., Tang, L.C. and Zhang, F.J., et al., (2006). Experimental study of polycarbonate's impact compression. Polymer Materials Science and Engineering 22: 165-168.

Johnson, G.R. and Cook, W.H., (1983). A constitutive model and data for metals subjected to large strains, high strain rates and high temperatures. Proceeding of the Seventh International Symposium on Ballistics, 541-547.

Le Nard, H. and Bailly, P., (2000). Dynamic behavior of concrete: the structural effects on compressive strength increase. Mechanics of Cohesive Frictional Materials 5: 491-510.

Marais, S.T., Tait, R.B. and Cloete, T.J. , et al., (2004). Material testing at high strain rate using the split Hopkinson pressure bar. Latin American Journal of Solids and Structures 1: 319-339.

Pang, B.J., Yang, Z.Q. and Wang, L.W., et al. (2011). Dynamic compression properties and constitutive model with strain rate effect of rubber material. Chinese Journal of High Pressure Physics 25: 407-415.

Qi, C.Z. and Qian, Q.H., (2003). Physical mechanism of dependence of material strength on strain rate for rock-like material. Chinese Journal of Rock Mechanics and Engineering 22: 177-181.

Qi, C.Z., Wang, M.Y. and Qian, Q.H., (2009). Strain-rate effects on the strength and fragmentation size of rocks. International Journal of Impact Engineering 36: 1355-1364.

Ren, X.T., Zhou, T.Q. and Zhong, F.P., et al. (2010). Experimental study for the dynamic mechanical behavior of granite. Journal of Experimental Mechanics 25: 723-730.

Sato, Y, Yoshida, M. and Nagayama, K., et al., (2008). Stress-strain relationships of polycarbonate over a wide range of strain rate, including a shock wave regime[J]. International Journal of Impact Engineering 35: 1778-1783.

Tu, Z.G. and Lu, Y., (2009). Evaluation of typical concrete material models used in hydrocodes for high dynamic response simulations. International Journal of Impact Engineering 36: 132-146.

Wang, Y.G. and Wang, C.L., (2011). Effect of structure characteristic parameters and strain rate on the compressive mechanical properties of aluminum foams. Acta Armamentarii 32: 106-111.

Zhang, H., Suo, T. and Li, Y.L., (2012). Mechanical behavior of a stainless steel material at elevated temperatures and high strain rates. Journal of Aeronautical Materials 32: 78-83.

Zhang, L.S., Huang, F.L. and Duan, Z.P., et al. (2011). The strain-rate effect of 7075Al yielding and a modified Johnson-Cook constitutive relation. Proceedings of Ninth Material Dynamics Conference, 143-148.

Zhou, H.L. and John, L., (2001). Strain rate effects on the thermomechanical behavior of polymers. International Journal of Solids and Structures 38: 3549-3562.

Zhou, X.Q., Hao, H. and Kuznetsov, V.A., et al. (2006). Numerical calculation of concrete slab response to blast loading. Transaction of Tianjin University (English edition) 12: 94-99. 